\title{
Oral manifestations of Type I Neurofibromatosis in a family
}

\author{
Mubeen Khan ${ }^{1}$, Neera Ohri ${ }^{2}$ \\ ${ }^{1}$ MDS. Professor and Head. Department of Oral Medicine and Radiology. Government Dental College and Research Institute. \\ Fort, Bangalore, Karnataka, India. \\ ${ }^{2}$ BDS (MDS). Post Graduate Student. Department of Oral Medicine and Radiology. Government Dental College and Research \\ Institute. Fort, Bangalore, Karnataka, India.
}

Correspondence:

Department of Oral Medicine and Radiology

Government Dental College and Research Institute

Fort, Bangalore-560002

Karnataka, India.

E-mail:neeraohri@gmail.com

Khan M, Ohri N. Oral manifestations of Type I Neurofibromatosis in a family. J Clin Exp Dent. 2011;3(5):e483-6

http://www.medicinaoral.com/odo/volumenes/v3i5/jcedv3i5p483.pdf

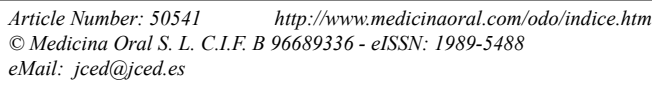

\begin{abstract}
Neurofibroma is a benign peripheral nerve sheath tumor. It is one of the most frequent tumors of neural origin and its presence is one of the clinical criteria for the diagnosis of neurofibromatosis type I (NF-I). Neurofibromatosis type $\mathrm{I}$ is an autosomal dominantly inherited disease due to an alteration in the long arm of chromosome 17. About $50 \%$ of NF-I patients have no family history of the disease. NF-I patients have skin lesions (café au lait spots and neurofibromas) as well as bone malformations and central nervous system tumors. Diagnosis is based on a series of clinical criteria. NF-I presents with certain definite oral manifestations which confers to the dentists a major responsibility for accurate diagnosis and report of the disease. The paper aims to highlight the role of an oral diagnostician in first recognition of NF-I in a family via manifestations in the oral cavity.
\end{abstract}

Key words: neurofibromatosis type I, oral manifestations. 


\section{Introduction}

Neurofibroma, a benign peripheral nerve sheath tumor arises from Schwann cells and perineural fibroblasts. Two clinical forms of neurofibromatosis have been described: peripheral- type I; and central- type II (1). Neurofibromatosis type I, also known as von Recklinghausen's disease, is a common neurocristopathy, with an estimated incidence of 1 in 3,000 live births. It is characterized by autosomal dominant inheritance with complete penetrance but variable expression. The tumor suppressor NF1gene has been identified on chromosome17q11.2 (2).

The frequency of oral manifestations is debated in the literature. Some authors report a frequency of $4-7 \%$ of cases (3), whereas others suggest that these manifestations are present in up to $72 \%$ of cases (4). The present paper focuses on oral manifestations of Neurofibromatosis type I in a family and the role of oral diagnostician in accurate and early diagnosis which is central to timely treatment and ultimate well being of the patient.

\section{Clinical Case}

Case 1: A 20-year-old female patient reported to Department of Oral medicine and radiology, Government Dental College and Research Institute, with a complain of pain in left maxillary tooth and swelling in left angle of mandible since one month. The physical examination disclosed multiple skin nodules distributed throughout the body, extensively involving the face and neck and were present since childhood. The diffuse brownish pigmentation was present on the back and neck. The family history revealed that mother, sister and brother had similar nodules on body. The extra-oral examination revealed flat nose, frontal bossing with palpable,

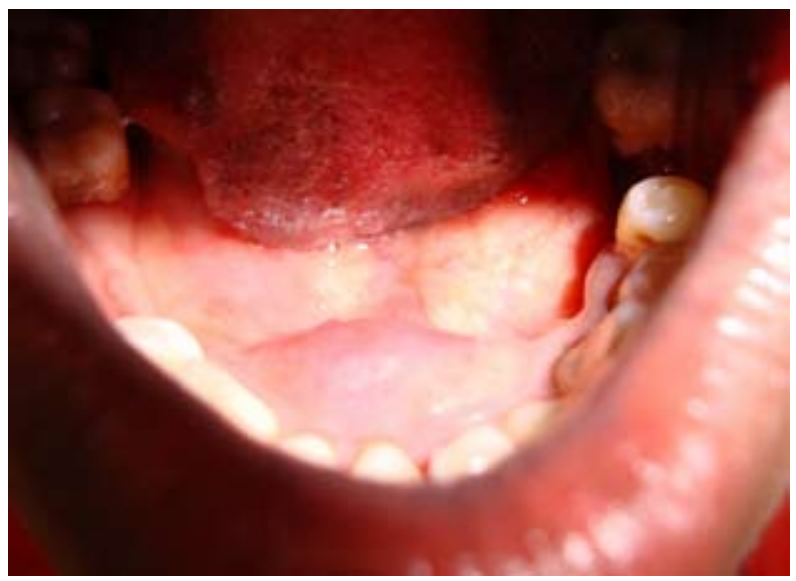

Fig. 1. intra-oral swelling present lingually in relation to lower anteriors.

tender and mobile bilateral submandibular lymph nodes. The ophthalomogical examination revealed presence of lisch nodules with everted eyelids. A localized swelling measuring about $2 \times 1.5 \mathrm{~cm}$ in dimensions with erythmatous surface and soft consistency was present on left angle of mandible. There was present diffuse brownish pigmentation of oral mucosa including tongue. Intraorally a swelling was noted lingually in relation to lower anterior teeth extending from attached gingival to floor of mouth (fig. 1.). The swelling was of normal mucosal color, almost $2.5 \mathrm{~cm}$ in diameter, non ulcerated, non tender, soft in consistency and had a sessile base. Recession with grade II mobility was present in relation to 31 . All the lower anterior teeth were vital and aspiration was negative. No odontogenic cause was found for extra-oral swelling and intraoral swelling. The patient was referred for dermatological opinion and extra oral swelling was

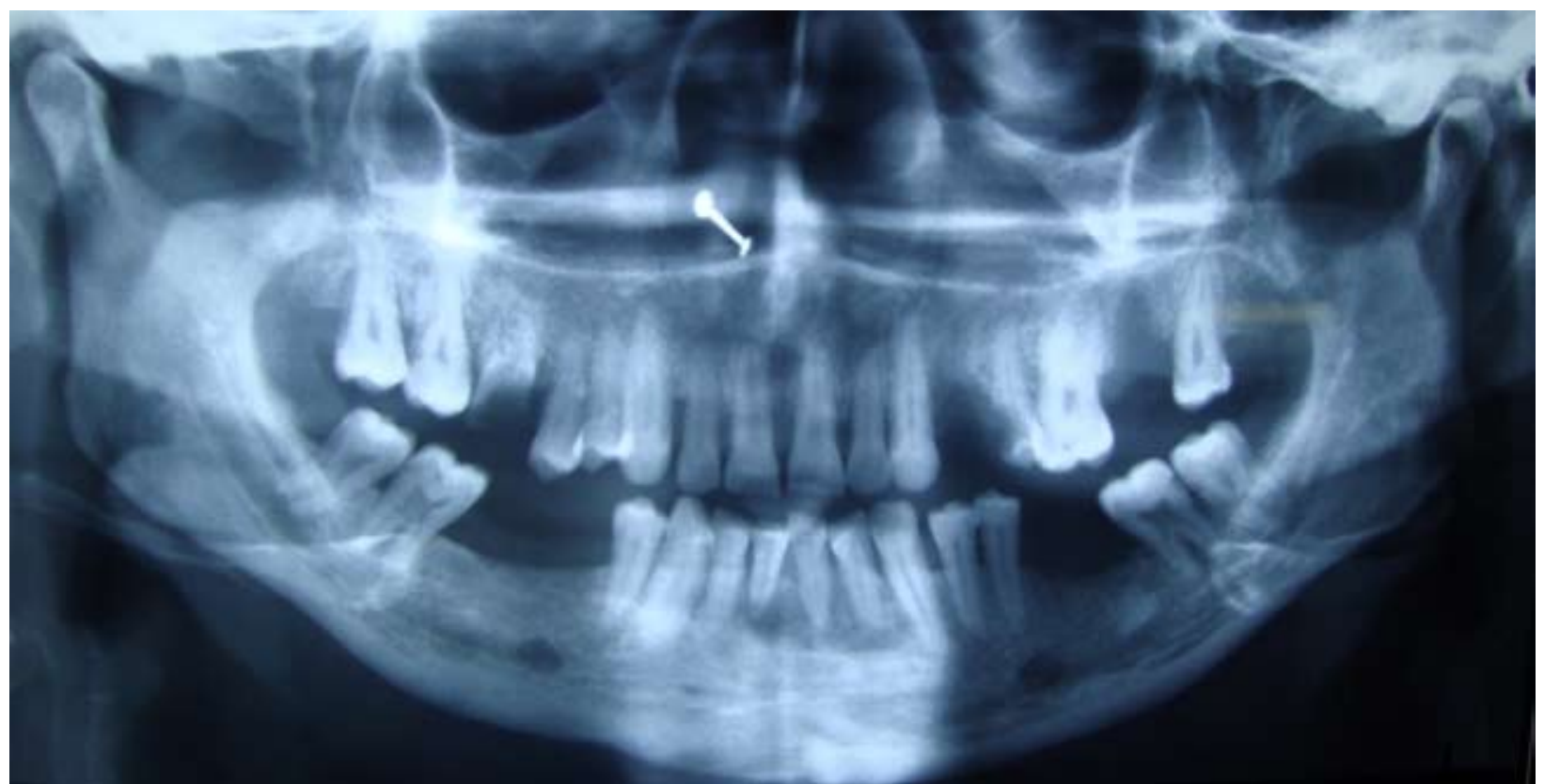

Fig. 2. Orthopantomograph showing widening of mandibular canals, mental foramen, deeepnening of sigmoid notch and shortening of ramus 


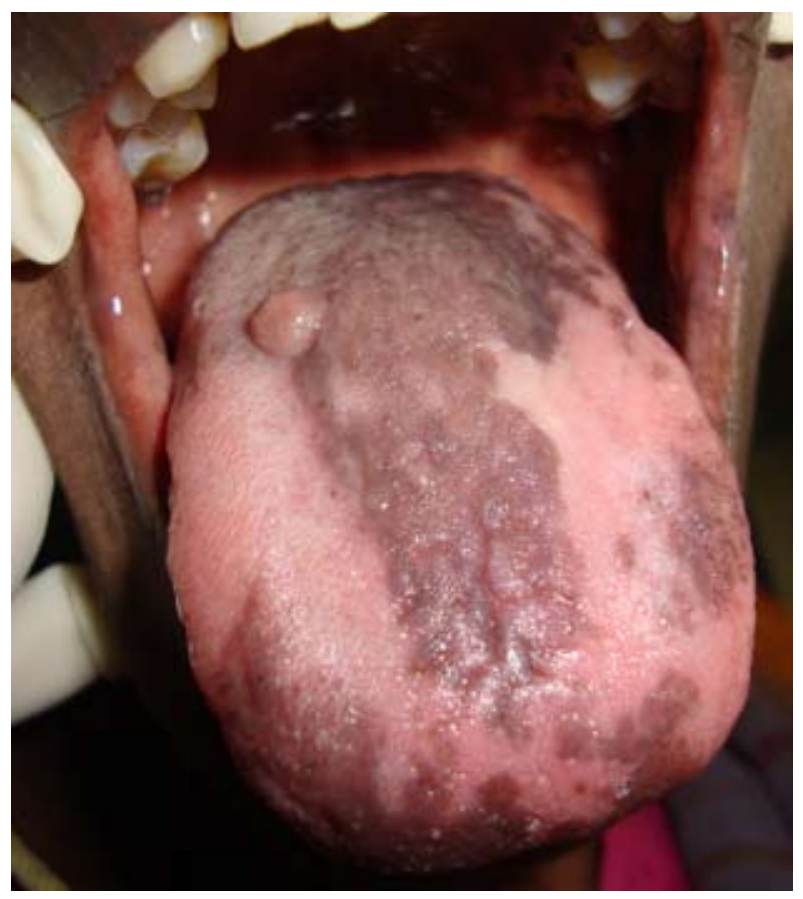

Fig. 3. a nodular lesion with sessile base present on tongue along with deep fissuring and brownish pigmentation

found to be secondarily infected neurofibroma and was put on analgesics and antibiotics. Hence the clinical diagnosis of Neurofibrotosis type I was given based on clinical criteria.

The orthopantomograph revealed diffuse radiolucency in periapical region in relation to 42,31 and 32 . There was widening of mandibular canals, enlarged mental foramen, deeepnening of sigmoid notch and shortening of ramus suggestive of neurofibromatosis. The enostosis in relation to missing 36 was also noted (fig. 2.). Hence the excisional biopsy of the intra-oral swelling was done. The histological picture was suggestive of neurofibroma. The H\&E stained sections revealed numerous elongated cells with wavy dark staining nuclei. The cells were seen associated with delicate strands of collagen fibers. Few cells are arranged in whorls and fascicles. Small amount of mucoid-like material was seen at areas. Few small neurites were also evident. Sections stained with toludine blue revealed mast cells.

Case 2: The elder sister of case I, aged 23 years had similar lesions on the body along with café-au-lait spots suggestive of type I neurofibromatosis. Intra-orally a nodular lesion with sessile base measuring about $1 \mathrm{~cm}$ in diameter along with deep fissures and brownish pigmentation was present on anterior dorsum of the tongue (fig. 3.). On palpation lesion was soft in consistency and non tender. No significant radiographic manifestations were found. The patient was counseled for excisional biopsy.

Case 3: The elder brother of case I, aged 29 years had multiple sessile and pedunclated lesions all over the body, with café- au- lait spots suggestive of type I neurofibromatosis. The patient had flat bridge of nose. Intraorally dorsum of tongue showed enlargement of fungiform papilla with patchy areas of depapillation and deep fissuring. No prominent radiographic manifestations were present.

\section{Discussion}

NF I is a multisystem harmartomatous disorder with protean expression of cutaneous, neurologic, skeletal, visceral, and ocular manifestations (2).Despite the advances of molecular biology, the diagnoses of NF I and NF II are still based on clinical criteria. The National Institutes of Health Consensus Development Conference has suggested clinical criteria diagnostic of NF I and NF II (5). Diagnosis of Neurofibromatosis Type I (NF I):

1. Six or more café au lait macules over $5 \mathrm{~mm}$ in greatest diameter in prepubertal individuals and over 15 $\mathrm{mm}$ in greatest diameter in postpubertal individuals

2. Two or more neurofibromas of any type or one plexiform neurofibroma

3. Freckling in the axillary or inguinal regions (Crowe's sign)

4. Optic glioma

5. Two or more Lisch nodules (iris harmartomas)

6. A distinctive osseous lesion such as sphenoid dysplasia or thinning of long bone cortex with or without pseudoarthrosis

7. A first-degree relative (parent, sibling, or offspring) with NF I by the above criteria

The criteria are met in an individual if two or more of the features listed are present.

Diagnosis of Neurofibromatosis Type II (NF II):

1. Bilateral masses of the eighth cranial nerve seen with appropriate imaging techniques (e.g., CT or MRI)

2. One relative in first-degree with NF II and either:

a) Unilateral mass of the eighth cranial nerve, or

b) Two of the following:

- Neurofibroma

- Meningioma

- Glioma

- Schwannoma

- Juvenile posterior subcapsular lenticular opacity

The criteria are met by an individual who satisfies condition 1 or 2 .

Neurofibromas are the hallmark of the NF I and usually appear during childhood or adolescence after the emergence of cafe' au lait spots. Our all the three cases had neurofibromas of the skin and cafe' au lait spots. According to Shapiro et al lesions in the oral soft tissues have been noted, with a reported prevalence up to $72 \%$ (4). The considerable difference of oral manifestations can be attributed to the heterogeneity of patients examined 
in specialized hospitals, and the differences in symptoms investigated and the methods of investigation applied. The neurofibromas in oral cavity most commonly involves the tongue. Other affected sites include lips, palate, buccal mucosa, gingiva, floor of the mouth or the pharynx (3). Neurofibromas of the tongue are nearly always nodular which was present in our case. Macroglossia and enlargement of filliform papillae may also be noted. One of our case showed additional finding of depapillation and deep fissuring of tongue. Oral localized neurofibromata present as discrete nodules of normal color and they are usually asymptomatic as was present in our case. Nevertheless, neurofibromata on or adjacent to the cranial nerves also affect motor function of the facial and hypoglossal nerves and sensory function of the trigeminal nerve. In our case, a localized neurofibroma was found in the gingiva, which is an uncommon localization. Shapiro et al. observed gingival neurofibromas in only $5 \%$ of all patients with NF I(4).Gingival lesions can be confused with periodontal disease or cause periodontal disease, because tissue growth impedes access to dental surfaces and is an obstacle to proper oral hygiene.

Oral radiographic findings unique to NF include lengthening, narrowing and rarefaction of coronoid and articular process, deepening of sigmoid notch, an enlarged mandibular canal, mandibular foramen and mental foramen. Other findings are Shortening of the ramus, notching of the inferior border of the mandible and even asymmetrically formed maxillary sinus (6). Osseous alterations can be the result of soft tissue tumor growing against or within bone, resulting in hypoplasia or resorption (3). The osseous changes in case I can be attributed to the soft tissue tumor growing against the bone.

A change in size of a pre-existing mass, compression, or infiltration of the adjacent structures indicate malignant degeneration(7).Thus, histological and clinical evaluation should be performed in order to choose the most appropriate treatment strategy for these patients. The partial or total surgical removal of tumors can be performed to solve aesthetic and functional problems, but it is preferable to wait for the end of growth to reduce the risk of re-occurrence. There is no indication to date that surgery favors malignant degeneration.

\section{Conclusion}

Occasionally, oral manifestations and oral tissue specimens may provide the opportunity to diagnose NF. The oral manifestations of NF are well-documented but may not be at the forefront of the clinician's mind in the differential diagnosis of intra-oral swelling. A thorough examination and trained eye will provide the opportunity to diagnose NF. Therefore, the oral diagnostician should be made aware of the oral manifestations of the NF which will help in timely diagnosis and treatment of this disorder.

\section{References}

1. Cunha KSG, Barboza EP, Dias EP and Oliveira FM. Neurofibromatosis type I with periodontal manifestation. A case report and literature review. Br Dent J. 2004;198:457-460.

2. Honavar SG, Singh AD, Shields CL, Shields JA and Eagle RC. Iris Melanoma in a patient with neurofibromatosis. Surv Ophthalmol. 2000; 45:231-236.

3. Geist JR, Gander DL, Stefanac SJ. Oral manifestations of neurofibromatosis types I and II. Oral Surg Oral Med Oral Pathol. 1992; 73:376-382.

4. Shapiro SD, Abramovitch K, Van Dis ML, Skoczylas LJ, Langlais $\mathrm{RP}$, Jorgenson RJ, et al. Neurofibromatosis: oral and radiographic manifestations. Oral Surg Oral Med Oral Pathol. 1984;58:493498.

5. Gutmann DH, Aylsworth A, Carey JC, Korf B, Marks J, Pyeritz $\mathrm{RE}$ et al. The diagnostic evaluation and multidisciplinary management of neurofibromatosis 1 and neurofibromatosis 2. JAMA. 1997;278:51-57.

6. Friedrich RE, Giese M, Schmelzle R, Mautner VF, Scheuer HA. Jaw malformations plus displacement and numerical aberrations of teeth in neurofibromatosis type 1: a descriptive analysis of 48 patients based on panoramic radiographs and oral findings. J Cranio Maxillofac Surg. 2003;31:1-9.

7. Vincent SD, Williams TP. Mandibular abnormalities in neurofibromatosis. Oral Surg Oral Med Oral Pathol.1983;55:253-258. 